\title{
Interacting dark sector with variable vacuum energy
}

\author{
Luis P. Chimento, ${ }^{1}$ Martín G. Richarte,${ }^{1}$ and Iván E. Sánchez García. ${ }^{1}$ \\ ${ }^{1}$ Departamento de Física, Facultad de Ciencias Exactas y Naturales, \\ Universidad de Buenos Aires and IFIBA, CONICET, \\ Ciudad Universitaria, Pabellón I, Buenos Aires 1428, Argentina
}

(Dated: October 22, 2013)

\begin{abstract}
We examine a cosmological scenario where dark matter is coupled to a variable vacuum energy while baryons and photons are two decoupled components for a spatially flat Friedmann-RobertsonWalker spacetime. We apply the $\chi^{2}$ method to the updated observational Hubble data for constraining the cosmological parameters and analyze the amount of dark energy in the radiation era. We show that our model fulfills the severe bound of $\Omega_{x}(z \simeq 1100)<0.009$ at the $2 \sigma$ level, so it is consistent with the recent analysis that includes cosmic microwave background anisotropy measurements from the Planck survey, the Atacama Cosmology Telescope, and the South Pole Telescope along with the future constraints achievable by the Euclid and CMBPol experiments, and fulfills the stringent bound $\Omega_{x}\left(z \simeq 10^{10}\right)<0.04$ at the $2 \sigma$ level in the big-bang nucleosynthesis epoch.
\end{abstract}

PACS numbers:

Keywords: nonlinear interaction, variable vacuum energy, early dark energy, dark matter

\section{INTRODUCTION}

The existence of dark matter and dark energy have been supported by many observations such as cosmic microwave background, power spectrum of clustered matter, and supernovae Ia data among other probes [1 3 . Even though a fundamental (microscopic) theory for describing the dark sector remains elusive, observations suggest that the dark matter is connected with the formation of the large-structure [1] whereas dark energy is causing the expansion of the Universe to speed up, rather than slow down.

To understand the hidden nature of the interacting dark sector [13], one has to confront these models with the observational data; the transfer of energy could alter the cosmic history leading to testable imprints in the Universe [7]. The amount of dark energy in the recombination epoch should fulfill the bound by $\Omega_{\mathrm{x}}(z \simeq$ $1100)<0.01$ at least [6]. Additionally, the presence of vacuum energy during big-bang nucleosynthesis (BBN) is well motivated both by considerations of dark energy as well as inflation, giving as a stringent bound $\Omega_{\mathrm{x}}(1 \mathrm{Mev})<0.21$ so that the model does not affect the $\mathrm{BBN}$ process and therefore the abundance of light elements such as ${ }^{4} \mathrm{He}$ [4], 5]. The Planck mission indicates that $\Omega_{\mathrm{x}}(z \simeq 1100)<0.009$ with a $95 \%$ C.L., whereas the joint analysis based on future surveys (Euclid $+\mathrm{CMBPol}$ ) will be able to constrain $\Omega_{\mathrm{x}}(z \simeq 1100)$ down to 0.00092 ; the joint analysis of the Euclid+Planck data will be less restrictive because it will lead to $\Omega_{\mathrm{x}}(z \simeq 1100)<0.0022$ [10].

Our goal is to investigate a universe with an interacting dark sector and two decoupled components. We constrain the cosmic set of parameters by using the updated Hubble data and the severe bounds reported by the Planck mission on early dark energy among other observational data.

\section{THE MODEL}

We consider a spatially flat homogeneous and isotropic universe described by Friedmann-RobertsonWalker (FRW) spacetime with a line element given by $d s^{2}=-d t^{2}+a^{2}(t)\left(d x^{2}+d y^{2}+d z^{2}\right)$, with $a(t)$ being the scale factor. The universe is filled with interacting dark matter and variable vacuum energy (VVE) plus decoupled baryonic matter and radiation components. The evolution of the FRW universe is governed by the Friedmann and conservation equations,

$$
\begin{gathered}
3 H^{2}=\rho_{\mathrm{t}}=\rho_{\mathrm{r}}+\rho_{\mathrm{b}}+\rho_{\mathrm{m}}+\rho_{\mathrm{x}}, \\
\dot{\rho}_{\mathrm{r}}+3 H \gamma_{\mathrm{r}} \rho_{\mathrm{r}}=0, \quad \dot{\rho}_{\mathrm{b}}+3 H \gamma_{\mathrm{b}} \rho_{\mathrm{b}}=0, \\
\dot{\rho}_{\mathrm{m}}+\dot{\rho}_{\mathrm{x}}+3 H\left(\gamma_{\mathrm{m}} \rho_{\mathrm{m}}+\gamma_{\mathrm{x}} \rho_{\mathrm{x}}\right)=0,
\end{gathered}
$$

where $H=\dot{a} / a$ is the Hubble expansion rate, and the equations of state for each species take a barotropic form $p_{\mathrm{i}}=\left(\gamma_{\mathrm{i}}-1\right) \rho_{\mathrm{i}}$. Then the constants $\gamma_{\mathrm{i}}$ indicate the barotropic index of each component being $\mathrm{i}=\{\mathrm{r}, \mathrm{b}, \mathrm{m}, \mathrm{x}\}$, so that $\gamma_{\mathrm{r}}=4 / 3, \gamma_{\mathrm{b}}=1$, and $\gamma_{\mathrm{x}}=0$, whereas $\gamma_{\mathrm{m}}$ will be estimated later on. So, $\rho_{\mathrm{x}}$ plays the role of a VVE, $\rho_{\mathrm{b}}$ represents a pressureless baryonic matter, $\rho_{\mathrm{r}}$ is a radiation component and $\rho_{\mathrm{m}}$ can be associated with dark matter.

Solving the linear algebraic system of Eq. (3) along with $\rho=\rho_{\mathrm{m}}+\rho_{\mathrm{x}}$, we acquire both dark component densities as functions of $\rho$ and $\rho^{\prime}$

$$
\rho_{\mathrm{m}}=-\frac{\gamma_{\mathrm{x}} \rho+\rho^{\prime}}{\gamma_{\mathrm{m}}-\gamma_{\mathrm{x}}}, \quad \rho_{x}=\frac{\gamma_{\mathrm{m}} \rho+\rho^{\prime}}{\gamma_{\mathrm{m}}-\gamma_{\mathrm{x}}},
$$

where we introduced the variable $\eta=\ln \left(a / a_{0}\right)^{3},^{\prime} \equiv$ $d / d \eta$. The baryons and photons are decoupled from the dark sector, so Eq. (2) leads to $\rho_{\mathrm{r}}=\rho_{\mathrm{r} 0} a^{-3 \gamma_{\mathrm{r}}}$ and $\rho_{\mathrm{b}}=\rho_{\mathrm{b} 0} a^{-3 \gamma_{\mathrm{b}}}$, respectively. In order to continue the 
analysis of the interacting dark sector, we take into account the exchange of energy in the dark sector through the term $3 H Q$ into Eq. (3), which we will tackle next. It is convenient to use $d \eta=3 H d t$ to obtain the balance equations in a simpler form:

$$
\rho_{\mathrm{m}}^{\prime}+\gamma_{\mathrm{m}} \rho_{\mathrm{m}}=-Q, \quad \rho_{\mathrm{x}}^{\prime}+\gamma_{\mathrm{x}} \rho_{\mathrm{x}}=Q .
$$

From Eqs. (4) and (5), we obtain the source equation [13] for the energy density $\rho$ of the dark sector

$$
\rho^{\prime \prime}+\left(\gamma_{\mathrm{m}}+\gamma_{\mathrm{x}}\right) \rho^{\prime}+\gamma_{\mathrm{m}} \gamma_{\mathrm{x}} \rho=Q\left(\gamma_{\mathrm{m}}-\gamma_{\mathrm{x}}\right)
$$

Here, the nonlinear interaction $Q$ between both dark components is $Q=\alpha \rho^{\prime} \rho$, with $\alpha$ being the coupling constant. This interaction was not examined in the literature before and gives rise to a scenario where VVE can be viewed as a variable cosmological constant 14.

By replacing the specific form of $Q$ into the source equation (6), it turns into a nonlinear second-order differential equation for the total energy density $\rho$. Inserting $\gamma_{\mathrm{x}}=0$ into the latter equation, one gets its first integral

$$
\rho^{\prime}=\gamma_{\mathrm{m}}\left[\frac{\alpha}{2} \rho^{2}-\rho+\mathcal{D}\right]
$$

where $\mathcal{D}$ is an integration constant. Plugging Eq. (7) into Eq. (4), we obtain that $\rho_{\mathrm{x}}=\left[\alpha \rho^{2} / 2+\mathcal{D}\right]=\Lambda$, so dark energy can be considered as a VVE provided at late times $\rho_{t} \simeq \rho=3 H^{2}$ and then $\Lambda \simeq\left(\alpha H^{4} / 2+\mathcal{D}\right)$ [14]. In order to get $\rho(a)$, we need to express the first-order nonlinear differential equation (7) as an integration by quadrature. Solving Eq. (77) under the condition $1>2 \alpha \mathcal{D}$ allows us to obtain the total energy density of the dark sector

$$
\rho=\frac{\mathcal{K}(1+\mathcal{R}) a^{-3 \gamma_{\mathrm{m}} \mathcal{R}}+\mathcal{R}-1}{\alpha\left[\mathcal{K} a^{-3 \gamma_{\mathrm{m}} \mathcal{R}}-1\right]},
$$

where $\mathcal{R}=\sqrt{1-2 \alpha \mathcal{D}}, \mathcal{K}$ is an integration constant. Using the present-density parameters $\Omega_{i 0}=\rho_{i 0} / 3 H_{0}^{2}$ and the flatness condition, $1=\Omega_{\mathrm{r} 0}+\Omega_{\mathrm{b} 0}+\Omega_{\mathrm{x} 0}+\Omega_{\mathrm{m} 0}$, we write the integration constants $\mathcal{K}$ and $\mathcal{D}$ in terms of density parameters:

$$
\begin{gathered}
\mathcal{K}=\frac{3 \alpha H_{0}^{2}\left(\Omega_{\mathrm{x} 0}+\Omega_{\mathrm{m} 0}\right)-(1-\mathcal{R})}{3 \alpha H_{0}{ }^{2}\left(\Omega_{\mathrm{x} 0}+\Omega_{\mathrm{m} 0}\right)-(1+\mathcal{R})}, \\
\mathcal{D}=3 H_{0}{ }^{2} \Omega_{\mathrm{x} 0}-\frac{\alpha}{2}\left[3 H_{0}{ }^{2}\left(\Omega_{\mathrm{x} 0}+\Omega_{\mathrm{m} 0}\right)\right]^{2} .
\end{gathered}
$$

The total energy density is given by

$$
\begin{gathered}
\rho_{\mathrm{t}}=3 H_{0}{ }^{2}\left(1-\Omega_{\mathrm{b} 0}-\Omega_{\mathrm{x} 0}-\Omega_{\mathrm{m} 0}\right) a^{-3 \gamma_{\mathrm{r}}}+3 H_{0}{ }^{2} \Omega_{\mathrm{b} 0} a^{-3} \\
+\frac{\mathcal{K}(1+\mathcal{R}) a^{-3 \gamma_{\mathrm{m}} \mathcal{R}}+\mathcal{R}-1}{\alpha\left[\mathcal{K} a^{-3 \gamma_{\mathrm{m}} \mathcal{R}}-1\right]}
\end{gathered}
$$

The Universe is dominated by radiation at early times. After this epoch pressureless baryonic matter dominates followed by an era dominated by dark matter when $\gamma_{\mathrm{m}} \mathcal{R} \simeq 1$, ending with a de Sitter phase at late times [cf. Eq. (10)]. To see that dark matter dominates the evolution of the Universe during a short period of time, we use energy density of the dark sector (8) and we find $\rho=(1 / \alpha)-(\mathcal{R} / \alpha)[1+x /(1-x)]$, where $x=\mathcal{K} a^{-3 \gamma_{\mathrm{m}} \mathcal{R}}$. When $x$ is considerably small, Eq. (8) leads to $\rho \simeq(1-\mathcal{R}) / \alpha-(2 \mathcal{R} / \alpha) x$ along with $\rho_{\mathrm{m}}=$ $-\rho^{\prime} / \gamma_{\mathrm{m}} \simeq\left(2 \mathcal{R} / \alpha \gamma_{\mathrm{m}}\right) x^{\prime}$. Using $x^{\prime}=-\mathcal{K} \gamma_{\mathrm{m}} \mathcal{R} a^{-3 \gamma_{\mathrm{m}} \mathcal{R}}$, we obtain that $\rho_{\mathrm{m}} \simeq-\left(2 \mathcal{R}^{2} \mathcal{K} / \alpha \gamma_{\mathrm{m}}\right) a^{-3 \gamma_{\mathrm{m}} \mathcal{R}}>0$ provided $\mathcal{K}<0$; such fact can be verified by using the best-fit values of the cosmological parameters found in the next section.

\section{OBSERVATIONAL HUBBLE DATA CONSTRAINTS}

We focus on the cosmological constraints on the parameter space of the interacting dark sector plus the decoupled radiation and baryonic components. The statistical analysis is based on the $\chi^{2}$ function of the Hubble data which is constructed as (see, e.g., Ref. [23])

$$
\chi^{2}(\theta)=\sum_{k=1}^{29} \frac{\left[H\left(\theta, z_{k}\right)-H_{\mathrm{obs}}\left(z_{k}\right)\right]^{2}}{\sigma\left(z_{k}\right)^{2}}
$$

where the $\theta$ denotes a set of parameters, $H_{\mathrm{obs}}\left(z_{k}\right)$ is the updated observational $H(z)$ data at the redshift $z_{k}$, and $\sigma\left(z_{k}\right)$ is the corresponding $1 \sigma$ uncertainty [15]. We will use data listed in Ref. [15] and the Hubble parameter at $z=0$ [22], commenting how the observational data were obtained [15]. The first compilation of nine measurements of the Hubble parameter as a function of the redshift in the range $0.1<z<1.75$ was used for constraining a dark energy model in Ref. 16. Stern et al. added two more observations in the redshift range $0.2<z<1.0$ [17], and another eight new high-accuracy estimates of $H(z)$ led to a sample of 19 observational $H(z)$ measurements, spanning almost 10 Gyr of cosmic time [18]. Further, Blake et al. obtained three data points by combining a baryon acoustic peak and an Alcock-Paczynski distortion from galaxy clustering in the WiggleZ Dark Energy Survey [19], whereas from the Sloan Digital Sky Survey Data Release 7 within a redshift window $0<z<0.4$, Zhang et al. obtained four new observational $H(z)$ data points [20]. Another two data points were added by considering the baryonic acoustic oscillations scale as a standard ruler in the radial direction [21]. Using Eqs. (9) and (10), one gets the Hubble parameter in terms of the redshift, $z=a^{-1}-1$, and the relevant cosmological parameters $\theta=\left(H_{0}, \Omega_{\mathrm{b} 0}, \Omega_{\mathrm{x} 0}, \Omega_{\mathrm{m} 0}, \alpha, \gamma_{\mathrm{m}}\right)$ as follows, $H(z, \theta)=\left[\rho_{\mathrm{t}} / 3\right]^{1 / 2}$.

The two-dimensional C.L. obtained with a standard $\chi^{2}$ function for two parameters is shown in Fig. (1), and the estimation of these parameters is briefly summarized in Table (II); reporting their corresponding marginal $1 \sigma$ error bars 24]. We find the best fit at $\left(\Omega_{\mathrm{b} 0}, \Omega_{\mathrm{x} 0}\right)=\left(0.059_{-0.107}^{+0.113}, 0.74_{-0.07}^{+0.07}\right)$ with 

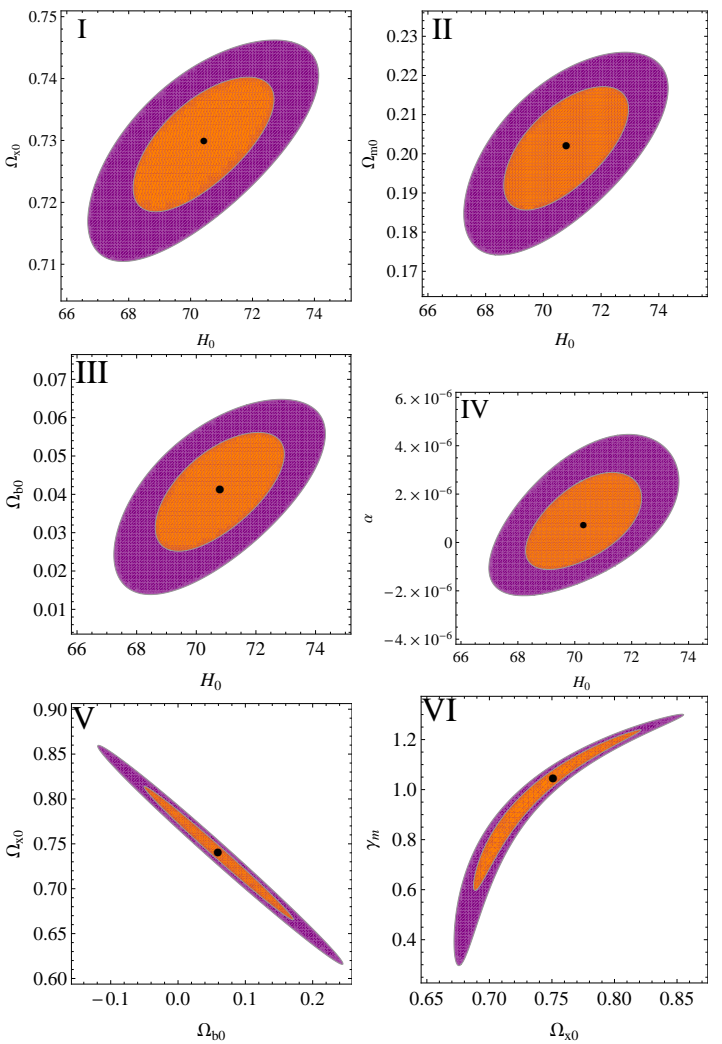

FIG. 1: Two-dimensional C.L. associated with $1 \sigma, 2 \sigma$ for different $\theta$ planes.

\begin{tabular}{|l|l|l|l|}
\hline \multicolumn{4}{|c|}{$2 D$ Confidence level } \\
\hline $\mathrm{N}$ & Priors & Bestfits & $\chi_{\mathrm{d} . \mathrm{o.f}}^{2}$ \\
\hline $\mathrm{I}$ & $\left(\Omega_{\mathrm{b} 0}, \Omega_{\mathrm{m} 0}, \alpha, \gamma_{\mathrm{m}}\right)=\left(0.051,0.219,10^{-6}, 1.010\right)$ & $\left(H_{0}, \Omega_{\mathrm{x} 0}\right)=\left(70.40_{-2.24}^{+2.31}, 0.73 \pm 0.01\right)$ & 0.725 \\
\hline $\mathrm{II}$ & $\left(\Omega_{\mathrm{b} 0}, \Omega_{\mathrm{x} 0}, \alpha, \gamma_{\mathrm{m}}\right)=\left(0.049,0.74,10^{-7}, 1.014\right)$ & $\left(H_{0}, \Omega_{\mathrm{m} 0}\right)=\left(70.79_{-2.19}^{+2.11}, 0.202_{-0.016}^{+0.015}\right)$ & 0.703 \\
\hline $\mathrm{III}$ & $\left(\Omega_{\mathrm{x} 0}, \Omega_{\mathrm{m} 0}, \alpha, \gamma_{\mathrm{m}}\right)=\left(0.74,0.21,10^{-7}, 1.014\right)$ & $\left(H_{0}, \Omega_{\mathrm{b} 0}\right)=\left(70.79_{-2.17}^{+2.18}, 0.041_{-0.016}^{+0.015}\right)$ & 0.703 \\
\hline $\mathrm{IV}$ & $\left(\Omega_{\mathrm{b} 0}, \Omega_{\mathrm{x} 0}, \Omega_{\mathrm{m} 0}, \gamma_{\mathrm{m}}\right)=(0.049,0.73,0.220,1.010)$ & $\left(H_{0}, \alpha\right)=\left(70.30_{-2.04}^{+2.05},\left[7.5_{-7.61}^{+7.1} \times 10^{-7}\right)\right.$ & 0.724 \\
\hline $\mathrm{V}$ & $\left(H_{0}, \Omega_{\mathrm{m} 0}, \alpha, \gamma_{\mathrm{m}}\right)=\left(69.04,0.20,10^{-7}, 1.036\right)$ & $\left(\Omega_{\mathrm{b} 0}, \Omega_{\mathrm{x} 0}\right)=\left(0.059_{-0.17}^{+0.113}, 0.74_{-0.07}^{+0.07}\right)$ & 0.753 \\
\hline $\mathrm{VI}$ & $\left(H_{0}, \Omega_{\mathrm{b} 0}, \Omega_{\mathrm{m} 0}, \alpha\right)=\left(68.5,0.043,0.205,10^{-8}\right)$ & $\left(\Omega_{\mathrm{x} 0}, \gamma_{\mathrm{m} 0}\right)=\left(0.77_{-0.06}^{+0.07}, 1.047_{-0.452}^{+0.192}\right)$ & 0.794 \\
\hline
\end{tabular}

TABLE I: We show the observational bounds for the 2D C.L. obtained in Fig. (1) by varying two cosmological parameters.

\begin{tabular}{|c|c|}
\hline Mission & Bound on $\left.\Omega_{x}\left[z \simeq 10^{3}\right)\right]$ \\
\hline Us & $\leq 0.000001$ \\
\hline Euclid & $<0.024$ \\
\hline CMBPol & $<0.0025$ \\
\hline SPT & $<0.02$ \\
\hline WMAP7 & $<0.018$ \\
\hline WMAP7+ACT & $<0.025$ \\
\hline WMAP7+SPT & $<0.013$ \\
\hline $\mathrm{WMAP} 7+\mathrm{SPT}+\mathrm{BAO}+\mathrm{SNe}$ & $<0.014$ \\
\hline Planck+WP & $<0.010$ \\
\hline Planck+WP+high L & $<0.009$ \\
\hline Planck+Euclid & $<0.0022$ \\
\hline CMBPol+Euclid & $<0.00092$ \\
\hline
\end{tabular}

TABLE II: Comparison of different estimations or simulations on the fraction of dark energy at early times.

$\chi_{\text {d.o.f }}^{2}=0.753$ by using the priors $\left(H_{0}, \Omega_{\mathrm{m} 0}, \alpha, \gamma_{\mathrm{m}}\right)=$ $\left(69.04 \mathrm{~km} \mathrm{~s}^{-1} \mathrm{Mpc}^{-1}, 0.20,10^{-7}, 1.036\right)$; the values of $\Omega_{\mathrm{b} 0}$ and $\Omega_{\mathrm{x} 0}$ agree with the data released by
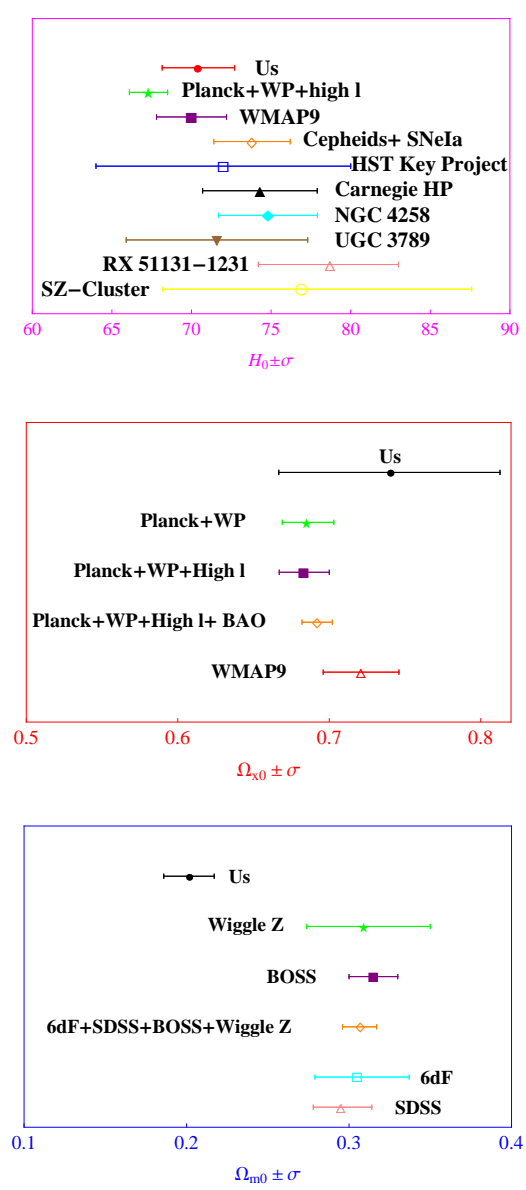

FIG. 2: Comparison of Hubble parameter, dark energy and dark matter amounts, with estimates of $\sigma$ errors, from a number of different methods.
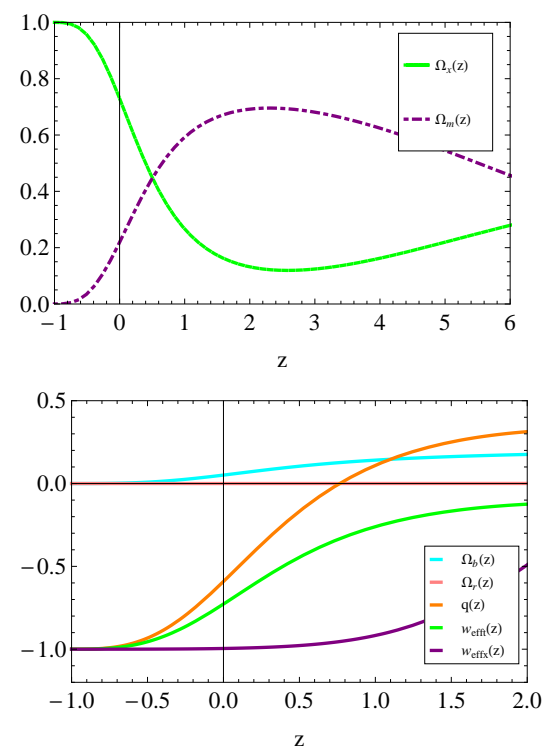

FIG. 3: Upper panel: Plot of $\Omega_{\mathrm{x}}$ and $\Omega_{\mathrm{m}}$ in terms of the redshift $z$. Lower panel: Plot $\Omega_{\mathrm{b}}, \Omega_{\mathrm{r}}, \mathrm{w}_{\mathrm{efft}}, \mathrm{w}_{\mathrm{effx}}$, and $q$ in terms of the redshift $z$. 
the Planck mission 2] and WMAP9 project 3] [see Fig. (2) ]. Indeed, Planck+WP data indicate that $\Omega_{\mathrm{x} 0}=0.685_{-0.016}^{+0.018}$ at a $68 \%$ C.L.; Planck $+\mathrm{WP}+$ high $\mathrm{L}$ data lead to $\Omega_{\mathrm{x} 0}=0.6830_{-0.016}^{+0.017}$ at $68 \%$ C.L. 2] [see Fig. (2)]. We get the best fit at $\left(H_{0}, \Omega_{\mathrm{m} 0}\right)=\left(70.79_{-2.19}^{+2.11} \mathrm{~km} \mathrm{~s}^{-1} \mathrm{Mpc}^{-1}, 0.202_{-0.016}^{+0.015}\right)$ along with $\chi_{\text {d.o.f }}^{2}=0.703<1$. The wiggleZ data give $\Omega_{\mathrm{m} 0}=0.309_{-0.035}^{+0.041}$ while the joint data $6 \mathrm{dF}+\mathrm{SDSS}+$ BOSS+ WiggleZ lead to $\Omega_{\mathrm{m} 0}=0.307_{-0.011}^{+0.010}$ at $68 \%$ C.L 2], showing a discrepancy on $\Omega_{\mathrm{m} 0}$ no bigger than $0.32 \%$ [see Figs. (2) and (3)]. The analysis leads to $\left(\Omega_{\mathrm{x} 0}, \gamma_{\mathrm{m}}\right)=\left(0.77_{-0.06}^{+0.07}, 1.047_{-0.452}^{+0.192}\right)$, pointing that the dark matter is not pressureless provided the barotropic index is greater than the unity [cf. Table (I)]. Regarding the Hubble parameter, we find that it varies over a wide range, $H_{0} \in\left[70.30_{-2.04}^{+2.05} ; 70.79_{-2.17}^{+2.18}\right] \mathrm{km} \mathrm{s}^{-1} \mathrm{Mpc}^{-1}$. Fitting the base $\Lambda$ cold dark matter model for the WMAP-9 data, it found $H_{0}=(70.0 \pm 2.2) \mathrm{kms}^{-1} \mathrm{Mpc}^{-1}$ at $68 \%$ C.L. 3], and agrees with our best estimations $H_{0}=$ $70.30_{-2.04}^{+2.05} \mathrm{kms}^{-1} \mathrm{Mpc}^{-1}$ at $68 \%$ C.L. In Fig. (2), we show bounds of $H_{0}$ that include the megamaser-based distance to NGC4258, SZ clusters, and others (see Ref. [2]). Figure (3) shows the decelerating parameter, density parameters, and equations of state with the redshift. Present-day value of $q(z=0) \in[-0.62 ;-0.59]$ as stated in the WMAP9 report [3]. The total equation of state, $\mathrm{w}_{\text {efft }}=-1+\sum_{\mathrm{j}} \gamma_{\mathrm{j}} \Omega_{\mathrm{j}}$, does not cross the phantom line neither the effective dark energy equation of state, and the same happens for dark energy effective equation of state, $\mathrm{w}_{\mathrm{effx}}=-\left[\alpha \rho \rho^{\prime}+\rho_{\mathrm{x}}\right] / \rho_{\mathrm{x}}$. Their values at $z=0$ vary over the intervals, $\mathrm{w}_{\mathrm{efft} 0} \in[-0.74,0.72]$ and $\mathrm{w}_{\mathrm{effx} 0} \in[-0.99,-0.97]$, respectively.

An interacting dark matter-VVE model has to be constrained with the physics behind recombination or big-bang nucleosynthesis epochs [7]. As is well known, the fraction of dark energy in the recombination epoch should fulfill the severe bound $\Omega_{\text {ede }}:=\Omega_{\mathrm{x}}(z \simeq 1100)<$ 0.01 [6]. The CMB measurements will put further constraints on early dark energy; the latest constraints on early dark energy come from the Planck+WP+high $\mathrm{L}$ data: $\Omega_{\text {ede }}<0.009$ at $95 \%$ C.L [2]. We found that $\Omega_{\mathrm{x}}\left(z \simeq 10^{3}\right) \in\left[10^{-6}, 10^{-5}\right]$, so our estimations satisfied the bound reported by the Planck mission [see Table (III)]. Further, the small-scale CMB temperature measurement from the SPT improves over WMAP7 alone by a factor of 3.5 [8], while WMAP7+SPT+BAO+SNe leads to $\Omega_{\text {ede }}<0.014$, and WMAP + SPT gives $\Omega_{\text {ede }}<0.013[9]$. Our value on $\Omega_{\mathrm{x}}(z \simeq 1100) \leq 10^{-6}$ at the $1 \sigma$ level is below the bounds achieved with the forecasting method applied to the Euclid project [10]; this survey will be able to constrain as $\Omega_{\text {ede }}<0.024$. We fulfill the bound reported from the joint analysis based on Euclid+CMBPol data, $\Omega_{\text {ede }}<0.00092$ [see Table (III)]. Our estimation on $\Omega_{\mathrm{x}}(z \simeq 1100)$ is much smaller than the bounds obtained by means of the standard Fisher matrix approach applied to the Euclid and CMBPol experiments [10], 12]. Around $z=10^{10}(\mathrm{BBN})$, we obtain that $\Omega_{\mathrm{x}} \in\left[10^{-34} ; 10^{-33}\right]$ at the $1 \sigma$ level, so the conventional BBN processes that occurred at a temperature of $1 \mathrm{Mev}$ are not spoiled [11].

\section{SUMMARY}

We have studied an interacting dark matter and VVE scenario along with decoupled baryons and photons components for a flat FRW universe; the new nonlinear interaction allowed for the dark mix to interpolate between a warm dark matter regime at early times, after the initial radiation regime, and end with a de Sitter phase.

The statistical analysis performed with the updated Hubble data [see Figs. (11)-(2) and Table (I)] allowed us to constrain the behavior of dark energy in the recombination era and compare it with the latest bounds coming from the Planck+WP+high $\mathrm{L}$ data, SPT, and ACT, among other experiments. We have found that $\Omega_{\mathrm{x}}\left(z \simeq 10^{3}\right) \in\left[10^{-6}, 10^{-5}\right]$, so our estimations satisfied the stringent bound reported by the Planck mission, $\Omega_{\text {ede }}<0.009$ at $95 \%$ C.L. [2] [see Table (III)] and agrees with the small-scale CMB temperature measurement from the SPT 8] or with the upper limit set by WMAP7+SPT+BAO+SNe data [9]. Further, the value $\Omega_{\mathrm{x}}\left(z \simeq 10^{3}\right) \simeq 10^{-6}$ obtained here will be consistent with the future constraints achievable by the Euclid and CMBPol experiments, 10], 12]. We also showed that dark energy around $z=10^{10}$ (BBN) fulfills the strong upper limit $\Omega_{\mathrm{x}}\left(z \simeq 10^{10}\right)<0.04$ at the $1 \sigma$ level 11 , so the standard BBN processes and the well-measured abundance of light elements are not disturbed.

\section{Acknowledgments}

The authors thank the anonymous referee for comments that greatly improved the clarity of the manuscript. L.P.C thanks U.B.A under Project No. 20020100100147 and CONICET under Project PIP No. 114-200801-00328. M.G.R and I.S.G are supported by CONICET.
[1] Y. Wang, "Dark energy", Wiley-vch Verlag GmbH and Co. KGaA, ISBN 978-527-40941-9 (2010); "Dark energy: Observational and theoretical approaches", edited by Pilar Ruiz-Lapuente, Cambridge University Press 2010.
[2] P. A. R. Ade et al, arXiv:1303.5076v1].

[3] G. Hinshaw et al., arXiv:1212.5226v3].

[4] E. W. Kolb and M. S. Turner, The Early Universe, Addison-Wesley, Redwood City, California (1990). 
[5] R.H. Cyburt, B.D. Fields, K. A. Olive and E. Skillman, Astropart. Phys. 23, 313 (2005).

[6] M.Doran and G. Robbers, JCAP 0606 (2006) 026, arXiv:astro-ph/0601544.

[7] L. P. Chimento, M. G. Richarte, Phys.Rev. D 84123507 (2011); L. P. Chimento, M. G. Richarte, Phys.Rev. D 85127301 (2012); L. P. Chimento and M. G. Richarte, Phys. Rev. D 86103501 (2012). L. P. Chimento, M. G. Richarte, Eur.Phys.J. C 73 (2013) 2352; L. P. Chimento, M. G. Richarte, Eur.Phys.J. C 73 (2013) 2497.

[8] Christian L. Reichardt, Roland de Putter, Oliver Zahn, Zhen Hou, arXiv:1110.5328.

[9] Z.Hou et al, [arXiv: 1212.6267]

[10] Lukas Hollenstein, Domenico Sapone, Robert Crittenden, Bjoern Malte Schaefer, JCAP 0904 (2009) 012.

[11] E.L. Wright, The Astrophysical Journal, 664 633-639, 2007.

[12] E. Calabrese, R. de Putter, D. Huterer, E. V. Linder, A. Melchiorri, Phys.Rev.D 83023011 (2011).

[13] L.P.Chimento, Phys.Rev.D81 043525 (2010).
[14] J. A. S. Lima, S. Basilakos and F. E. M. Costa, Phys. Rev. D 86103534 (2012); S. Basilakos, M. Plionis and J. Solà, Phys. Rev. D 803511 (2009).

[15] K. Liao, Z. Li, J. Ming, Z. H. Zhu, Phys. Lett. B 718 (2013) 1166-1170.

[16] J. Simon, L. Verde and R. Jimenez, Phys. Rev. D 71 123001 (2005) astro-ph/0412269. L. Samushia and B. Ratra, Astrophys. J. 650, L5 (2006).

[17] Stern D., Jimenez R., Verde L., et al., 2010, JCAP, 2, 8.

[18] M. Moresco, et al., JCAP 1208 (2012) 006.

[19] C. Blake et al., MNRAS, 425 (2012) 405.

[20] T. Zhang et al., 2012, arXiv:1207.4541,

[21] E. Gaztañaga, A. Cabré, and L. Hui. MNRAS, 399 (2009) 1663.

[22] Riess, A. G., et al. 2011, ApJ, 730, 119.

[23] Press, W.H., et al., Numerical Recipes in C. Cambridge University Press, Cambridge (1997).

[24] D. S. Sivia and J. Skilling, Data Analysis: A Bayesian Tutorial, Oxford University Press Inc., 2006. 